\title{
RELAÇÕES PRELIMINARES DA GEOMETRIA HIDRÁULICA REGIONAL PARA RIOS DAS REGIÕES OESTE E SUDOESTE DO ESTADO DO PARANÁ
}

\author{
OSCAR VICENTE QUINONEZ FERNANDEZ \\ Universidade Estadual do Oeste do Paraná
}

\section{Introdução}

O conceito de geometria hidráulica de canais fluviais, proposta por Leopold \& Maddock (1953), constitui umas das primeiras aplicações da análise quantitativa em geomorfologia fluvial (RHOADS, 1992). A geometria hidráulica descreve a relação entre variáveis dependentes como largura superficial, profundidade média e velocidade do fluxo e variáveis independentes como área da bacia ou vazão, e são expressas mediante funções potenciais. Informações detalhadas sobre aspectos teóricos e metodológicos da geometria hidráulica são encontradas nos trabalhos de Knighton (1975), Park (1977), Thornes (1977), Ferguson (1986), entre outros. No Brasil, destacam-se os trabalhos de Christofoletti (1976, 1981), Latrubesse e Aquino (1998) e Aquino et al. (2005).

A relação entre os elementos geométricos do canal fluvial medidos em nível de margens plenas (largura, profundidade e capacidade do canal) com a área da bacia hidrográfica foi denominada por Dunne e Leopold (1978) de curvas regionais ou geometria hidráulica regional. As curvas regionais constituem um método gráfico que ilustra a relação matemática entre a área de drenagem e os elementos da geometria hidráulica numa determinada província fisiográfica (HARMAN et al., 1999; SWEET e GERATZ, 2003). Após o trabalho pioneiro de Dunne e Leopold (1978), outros pesquisadores desenvolveram curvas regionais para diferentes regiões fisiográficas dos Estados Unidos (WHITING et al., 1999; MOODY e ODEM, 1999; HARMAN et al., 1999; WHITE, 2001; CASTRO e JACKSON，2001; McCANDLESS，2003; SWEET e GERATZ，2003; CINNOTO, 2003; POWELL et al., 2004; EMMERT, 2004; MESSINGER e WILEY, 2004; SHERWOOD e HUITGER, 2005; KEATON et al., 2005). 


\section{Conceito de vazão dominante e definição do nível de margens plenas}

As variáveis dependentes da geometria hidráulica regional são medidas em nível de margens plenas. Os pesquisadores postulam que as dimensões físicas dos canais aluviais são produzidas por uma categoria de vazão que é muito efetiva no transporte de sedimentos. Esta descarga teórica, denominada de vazão dominante (dominant discharge), se mantida constante ao longo de um período de tempo, produziria as mesmas características morfológicas que são geradas por uma ampla variedade de descargas que ocorre continuamente nos cursos de água (INGLIS, 1949). O conceito de descarga dominante, profundamente enraizado na geomorfologia fluvial e na engenharia hidráulica (PICKUP e WARNER, 1976), foi discutido por Benson e Thomas (1966), Pickup e Rieger (1979), Andrews (1980) e Carling (1988), entre outros.

Os pesquisadores adotaram vários critérios para representar na prática o conceito da descarga dominante. De acordo com Pickup e Warner (1976), a descarga dominante pode ser materializada por três categorias de descargas: descarga efetiva, descarga com determinado intervalo de recorrência e descarga de margens plenas. A descarga efetiva é representada pelo fluxo que transporta o maior volume de sedimentos (ANDREWS, 1980; NASH, 1994; EMMETT e WOLMAN, 2001; CASTRO e JACKSON, 2001). A descarga dominante também pode ser estimada por métodos estatísticos. Wolman e Leopold (1957) estimaram que a descarga dominante tem recorrência de 1 a 2 anos. Hickin (1968), Dury $(1973,1976)$ e Riley (1976) adotaram a vazão de 1,58 anos de recorrência como descarga dominante. Pickup e Warner (1976) descobriram que o período de recorrência da descarga dominante no rio Cumberland (Austrália) pode variar de 4 a 10 anos. Williams (1978) determinou que $75 \%$ dos 51 cursos d'água analisados apresentaram vazões dominantes entre 1,03 e 5,0 anos de recorrência. Comparando os resultados de inúmeros trabalhos, Leopold (1994) concluiu que, para a maioria dos pesquisadores, o período de retorno da descarga dominante é de 1,0 a 2,5 anos.

A descarga de margens plenas, por sua vez, é definida como a descarga líquida que preenche o canal ao nível da planície de inundação ativa (WOLMAN e LEOPOLD, 1957). Esta planície é definida como uma superfície plana adjacente ao canal fluvial, modelada pela ação erosiva ou deposicional do fluxo das cheias e inundada pelo menos uma vez a cada três anos. Por outro lado, Williams (1978) define a descarga de margens 
plenas como a vazão que preenche o canal até atingir o topo da margem. O nível de margens plenas demarca o limite entre os processos fluviais que modelam o canal e os que constroem a planície de inundação e constitui o principal parâmetro em projetos de restauração de ambientes fluviais (ROSGEN, 1994).

Para definir o nível de margens plenas, seguindo a proposta de Wolman e Leopold (1957), é necessária a identificação da planície aluvial ativa. A detecção desta superfície pode ser difícil até em rios com planície de inundação (NAVRATIL et al., 2006). Por essa razão são utilizados diversos critérios morfológicos, sedimentológicos e botânicos para definir o nível de margens plenas. Williams (1978), num trabalho de revisão bibliográfica, elencou 11 critérios e seus respectivos proponentes:

1) A superfície do fundo de vale (NIXON, 1959; WOODYER, 1968; KELLERHALS et al., 1972; DURY, 1973);

2) Nível da planície de inundação ativa (WOLMAN e LEOPOLD, 1957; LEOPOLD e SKIBITZKE, 1967; EMMETT, 1975);

3) Nível do patamar inferior (SCHUMM, 1960, BRAY, 1972);

4) Nível do patamar intermediário (WOODYER, 1968);

5) Nível do patamar superior (KILPATRICK e BARNES Jr., 1964);

6) Nível médio das partes mais altas nas barras de canal (WOLMAN e LEOPOLD, 1957; HICKIN, 1968; LEWIS e McDONALD, 1973);

7) Nível do limite inferior da vegetação perene (SCHUMM, 1960; NUNNALLY, 1967; BRAY, 1972; LEOPOLD, 1994; RADECKIPAWLIK, 2002; WOHL at al. 2004; WOHL e WILCOX, 2005);

8) Nível do limite superior de depósitos arenosos (NUNNALLY, 1967; LEOPOLD e SKIBITZKE, 1967);

9) Nível com a relação mínima da razão largura/profundidade (WOLMAN, 1955; HARVEY, 1969; PICKUP e WARNER, 1976);

10) Nível correspondente ao primeiro máximo no índice de Riley (bench index, BI) (RILEY, 1972); e

11) Nível correspondente ao brusco aumento da relação área da seção transversal e largura do canal (WILLIAMS, 1978).

Nos vales íngremes e confinados das áreas montanhosas, a planície aluvial ativa é pouco desenvolvida ou inexiste. Nestas situações, os critérios citados acima dificilmente podem ser aplicados e são adotados 
referencias como níveis de escavação nas margens (MONTGOMERY e GRAN, 2001), presença de vegetação perene (RADECKI-PAWLIK, 2002) e depósitos de detritos lenhosos e mudanças na granulometria dos sedimentos em depósitos marginais (WOHL e WILCOX, 2005).

\section{Objetivo e Área de Estudo}

O objetivo deste trabalho é estimar as relações da geometria hidráulica regional para os rios que drenam as regiões Oeste e Sudoeste do Paraná (Figura 1).

As regiões em apreço são constituídas por rochas basálticas de idade cretácea (Formação Serra Geral) (NARDY et al., 2002) e estão inseridas no terceiro planalto paranaense, localizado na porção central da bacia sedimentar do Paraná. A origem dos planaltos da bacia do Paraná está relacionada com a evolução da Plataforma Brasileira. A ascensão epirogenêtica da Plataforma, ocorrida no Plioceno e Pleistoceno, soergueu rochas paleozóicas e mesozóicas para altitudes superiores a $1.000 \mathrm{~m}$ (ALMEIDA, 1966). Esta forte elevação submeteu a área de estudo a um continuo processo de dissecação. A precipitação acumulada anual varia de $1.600 \mathrm{~mm}$ (na região Oeste) a $1.900 \mathrm{~mm}$ (na região Sudoeste) e o clima, na classificação de Köppen, é do tipo Cfa, subtropical úmido, mesotérmico, verões quentes, geadas menos freqüentes e concentração de chuvas nos meses de verão (IAPAR, 1994). 


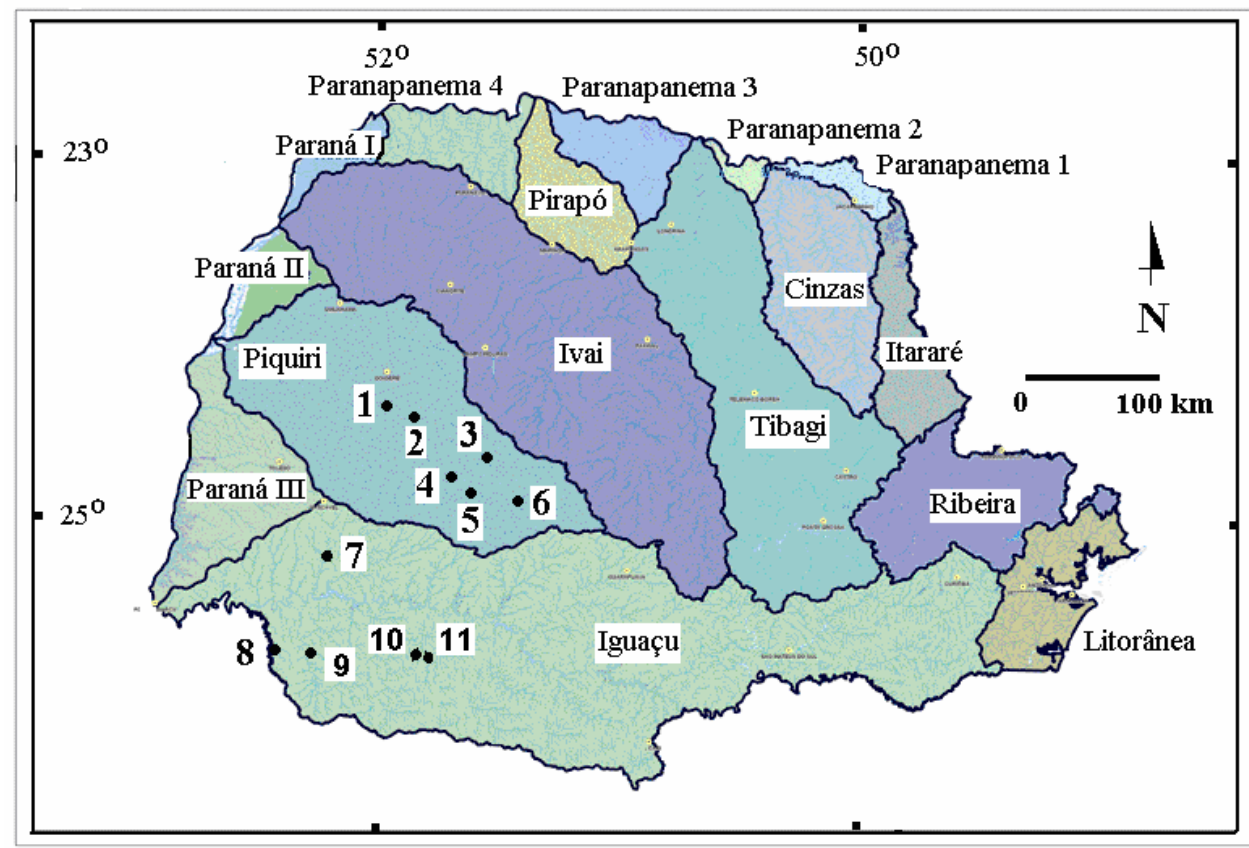

Figura 1: Localização das estações fluviométricas. Bacia do Piquiri: 1=Porto Novo II; 2=Ponte do Piquiri ; 3=Balsa do Cantu; 4=Foz do Cantu; 5=Porto Guarani e 6=Guampara. Bacia do Iguaçu: 7=São Sebastião; 8=Muniz, 9=Ponte do Capanema; 10=Balsa do Santana e 11=Águas do Verê. (FONTE: Suderhsa)

\section{Material e método}

Neste trabalho foram analisadas informações hidrométricas e morfológicas coletadas em estações hidrométricas implantadas nas bacias dos rios Piquiri e Iguaçu e operadas pela Suderhsa (Superintendência de Desenvolvimento de Recursos Hídricos e Saneamento Ambiental), órgão dependente da Secretária de Meio Ambiente do Estado do Paraná (Figura 1, Tabela 1).

Nas estações hidrométricas, o nível de margens plenas foi definido a partir de observações em campo, tendo como critério o limite superior dos depósitos de diques marginais, cuja cota foi obtida mediante levantamento topográfico convencional, tendo como referencias os RN`s das estações. A partir da definição do nível de margens plenas, foi estimada a vazão correspondente mediante as relações matemáticas entre a cota e a vazão 
das cheias (Tabela 1). Para obter relações mais acuradas, foram selecionadas estações hidrométricas com séries históricas superior a 15 anos. As equações foram obtidas a partir dos dados de vazão das cheias (maior vazão diária anual) e a respectiva cota, disponíveis no site da Agência Nacional de Águas (ANA) (http://hidroweb.ana.gov.br). As dimensões do canal em nível de margens plenas (largura, profundidade e capacidade do canal) foram calculadas para cada estação a partir de perfis transversais levantados pela Suderhsa.

\begin{tabular}{|c|c|c|c|c|c|}
\hline $\begin{array}{c}\text { Estação/ } \\
\text { Código (ANA) }\end{array}$ & $\begin{array}{l}\text { Coordenadas } \\
\text { Geográficas }\end{array}$ & Rio & $\begin{array}{l}\text { Área } \\
\left(\mathrm{km}^{2}\right)\end{array}$ & $\begin{array}{l}\text { Período } \\
\text { estudado }\end{array}$ & $\begin{array}{l}\text { Vazão das cheias, Q } \\
\left(\mathrm{m}^{3} / \mathrm{s}\right) \text { em função da } \\
\text { cota da cheia, } \mathrm{C}(\mathrm{cm}) \text {. }\end{array}$ \\
\hline $\begin{array}{l}\text { Balsa do Santana } \\
\quad 65955000\end{array}$ & $\begin{array}{lll}25^{\circ} & 54^{\prime} & 54^{\prime \prime} \mathrm{S} \\
52^{\circ} & 50^{\prime} & 59^{\prime \prime} \mathrm{W}\end{array}$ & Santana & 1.720 & $\begin{array}{l}1957- \\
2001\end{array}$ & $\begin{array}{l}Q=0,0201 . C^{1,7785} \\
R^{2}=0,996\end{array}$ \\
\hline $\begin{array}{c}\text { Muniz } \\
65990550 \\
\end{array}$ & $\begin{array}{ccc}25^{\circ} & 44^{\prime} & 83^{\prime \prime} \mathrm{S} \\
53^{\circ} & 50^{\prime} & 49 \prime \\
\end{array}$ & $\begin{array}{c}\text { Santo } \\
\text { Antonio }\end{array}$ & 969 & $\begin{array}{l}1986- \\
2004\end{array}$ & $\begin{array}{l}\mathrm{Q}=0,6895 \cdot \mathrm{C}-151,19 . \\
\mathrm{R}^{2}=0,990\end{array}$ \\
\hline $\begin{array}{c}\text { Ponte do Capanema } \\
65981500\end{array}$ & $\begin{array}{l}25^{\circ} 46^{\prime} 05^{\prime \prime} \mathrm{S} \\
53^{\circ} 36^{\prime} 42^{\prime \prime} \mathrm{W}\end{array}$ & $\begin{array}{c}\text { Capanem } \\
\text { a }\end{array}$ & 1.740 & $\begin{array}{l}1977- \\
2005\end{array}$ & $\begin{array}{l}\mathrm{Q}=0,4977 . \mathrm{C}-94,60 . \\
\mathrm{R}^{2}=0,999 .\end{array}$ \\
\hline $\begin{array}{c}\text { São Sebastião } \\
65979000\end{array}$ & $\begin{array}{l}25^{\circ} 27^{\prime} 37^{\prime \prime} \mathrm{S} \\
53^{\circ} 31^{\prime} 43^{\prime \prime} \mathrm{W}\end{array}$ & Andrada & 1.309 & $\begin{array}{l}1977- \\
2005 \\
\end{array}$ & $\begin{array}{l}\mathrm{Q}=0,758 . \mathrm{C}-133,92 . \\
\mathrm{R}^{2}=0,993\end{array}$ \\
\hline $\begin{array}{c}\text { Águas do Verê } \\
65966000\end{array}$ & $\begin{array}{lll}25^{\circ} & 46 & 37^{\prime \prime} \mathrm{S} \\
52^{\circ} & 55^{\prime} & 58^{\prime \prime} \mathrm{W}\end{array}$ & Chopim & 6.696 & $\begin{array}{l}1957- \\
2005\end{array}$ & $\begin{array}{l}\mathrm{Q}=0,0203 \cdot \mathrm{C}^{1,9309} \\
\mathrm{R}^{2}=0,991\end{array}$ \\
\hline $\begin{array}{c}\text { Balsa do Cantu } \\
64775000\end{array}$ & $\begin{array}{l}24^{\circ} 44^{\prime} 55^{\prime \prime} \mathrm{S} \\
52^{\circ} 42^{\prime} 10^{\prime \prime} \mathrm{W}\end{array}$ & Cantu & 2.513 & $\begin{array}{l}1968- \\
2005\end{array}$ & $\begin{array}{l}\mathrm{Q}=2,813 \cdot \mathrm{C}-765,54 \\
\mathrm{R}^{2}=0,990\end{array}$ \\
\hline $\begin{array}{l}\text { Guampará } \\
64764000\end{array}$ & $\begin{array}{c}24^{\circ} 58^{\prime} 59^{\prime \prime} \mathrm{S} \\
52^{\circ} 16^{\prime} 59^{\prime \prime} \mathrm{W}\end{array}$ & Piquiri & 1.703 & $\begin{array}{l}1985- \\
2001\end{array}$ & $\begin{array}{l}\mathrm{Q}=0,4905 \cdot \mathrm{C}+300,73 \\
\mathrm{R}^{2}=0,992\end{array}$ \\
\hline $\begin{array}{c}\text { Porto Guarani } \\
64771500\end{array}$ & $\begin{array}{c}24^{\circ} 51 ' 58^{\prime \prime} \mathrm{S} \\
52^{\circ} 45^{\prime} 46^{\prime \prime} \mathrm{W}\end{array}$ & Piquiri & 4.223 & $\begin{array}{l}1987- \\
2005\end{array}$ & $\begin{array}{l}\mathrm{Q}=0,0222 \cdot \mathrm{C}^{1,6596} \\
\mathrm{R}^{2}=0,999\end{array}$ \\
\hline $\begin{array}{c}\text { Foz do Cantu } \\
64776100\end{array}$ & $\begin{array}{c}24^{\circ} 45^{\prime} 7^{\prime \prime} \mathrm{S} \\
52^{\circ} 52^{\prime} 36^{\prime \prime} \mathrm{W}\end{array}$ & Piquiri & 7.690 & $\begin{array}{l}1987- \\
2005\end{array}$ & $\begin{array}{l}Q=0,0029 \cdot C^{2,161} \\
R^{2}=0,999\end{array}$ \\
\hline $\begin{array}{l}\text { Ponte do Piquiri } \\
64795000\end{array}$ & $\begin{array}{l}24^{\circ} 33^{\prime} 31^{\prime \prime} \mathrm{S} \\
53^{\circ} 07^{\prime} 45^{\prime \prime} \mathrm{W}\end{array}$ & Piquiri & 11.303 & $\begin{array}{l}1978- \\
2005 \\
\end{array}$ & $\begin{array}{l}\mathrm{Q}=6,175 \cdot \mathrm{C}-1139,9 \\
\mathrm{R}^{2}=0,997\end{array}$ \\
\hline $\begin{array}{c}\text { Novo Porto } 2 \\
64799500 \\
\end{array}$ & $\begin{array}{l}24^{\circ} 22^{\prime} 41^{\prime \prime} \mathrm{S} \\
53^{\circ} 09^{\prime} 45^{\prime \prime} \mathrm{W} \text {. }\end{array}$ & Piquiri & 12.124 & $\begin{array}{l}1978- \\
2005\end{array}$ & $\begin{array}{l}Q=7,532 \cdot C-1853,4 \\
R^{2}=0,998\end{array}$ \\
\hline
\end{tabular}

Tabela 1: Dados básicos das estações fluviométricas selecionadas nas bacias dos rios

Piquiri e Iguaçu (FONTE: Suderhsa). 


\section{Resultados e Discussões}

No terceiro planalto paranaense, a maior parte da rede de drenagem encontra-se encaixada na superfície de fundo de vale. Por essa razão, os critérios baseados em feições morfológicas e geométricas mostraram-se incapazes para definir o nível de margens plenas na área de estudo. Perante esta situação, Fernandez e Bortoluzzi (2008) optaram pela escolha de marcos sedimentológicos. Dentre os critérios sedimentológicos listados por Williams (1978), os autores escolheram o oitavo critério (Nível do limite superior de depósitos arenosos) por constituir feições deposicionais comuns tanto em margens com superfícies horizontais e baixas como em margens com superfícies declivosas e altas. Nas primeiras, os depósitos arenosos constituem diques marginais, que são feições identificadas com maior facilidade. No segundo tipo de margens, os sedimentos arenosos formam cordões longitudinais depositados em vários níveis, sendo neste caso selecionado como referencia as acumulações mais proeminentes.

Com base no critério acima exposto, foi definido o nível de margens plenas nas estações fluviométricas selecionadas. A partir deste nível, foram estimadas a vazão, largura, profundidade e capacidade do canal. Estas variáveis, consideradas como dependentes, foram confrontadas com a área da bacia correspondente, definida como variável independente. Como resultado foram obtidas as relações da geometria hidráulica regional ou curvas regionais para rios das regiões Oeste e Sudoeste do Paraná (Figura 2). Os coeficientes de correlação $\left(R^{2}\right)$ calculados mostram fortes associações entre a área da bacia e a vazão $(0,94)$, largura $(0,89)$ e capacidade de canal $(0,83)$. $\mathrm{O}$ valor de $\mathrm{R}^{2}$ para a relação entre área da bacia e a profundidade forneceu uma correlação moderada $(0,49)$. 


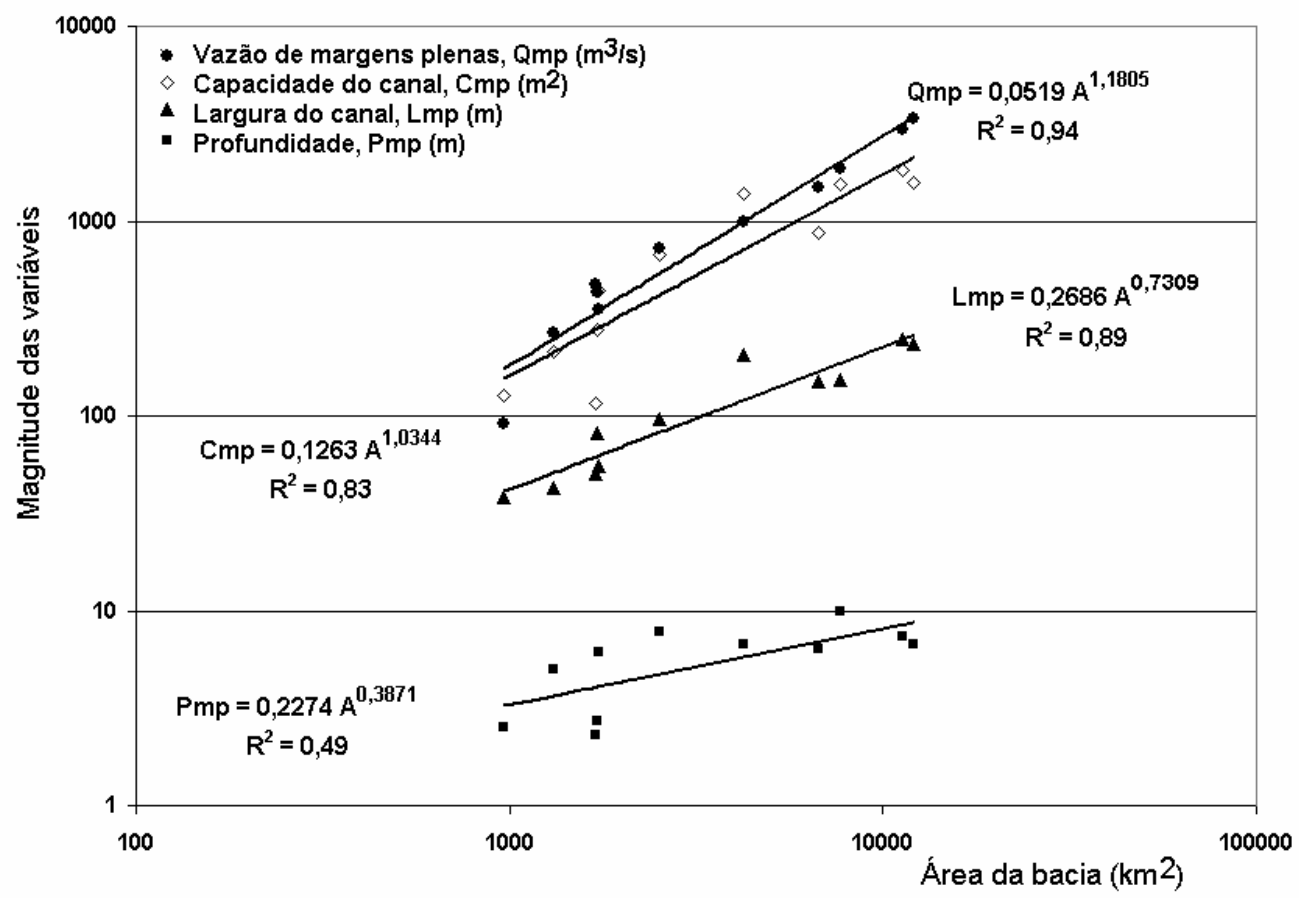

Figura 2 : Relações da geometria hidráulica regional em estações hidrométricas das regiões Oeste e Sudoeste do Paraná

As equações permitem estimar, de maneira razoável, a vazão e as dimensões do canal em nível de margens plenas para pontos de interesse sem estação fluviométrica, tendo somente como variável independente a área da bacia. As informações obtidas podem orientar técnicos em projetos de restauração de canais e direcionar trabalhos de avaliação das condições de degradação em ambientes fluviais

\section{Conclusões}

As relações da geometria hidráulica regional mostraram altos valores de coeficiente de correlação $\left(\mathrm{R}^{2}\right)$, que oscilam entre 49 e 94\%. Tais resultados foram obtidos em estações com áreas de drenagem que variam de 969 a $12.000 \mathrm{~km}^{2}$, instaladas nas regiões Oeste e Sudoeste do Paraná e inseridas em contextos geológicos e climáticos similares. Os significativos valores de $\mathrm{R}^{2}$ indicam que critérios sedimentológicos podem ser utilizados 
como referência na identificação do nível de margens plenas nos rios do planalto basáltico da bacia do Paraná.

\section{Agradecimentos}

O autor agradece à Agência Nacional de Águas (ANA), à Suderhsa (pela disponibilidade de dados hidrométricos/morfológicos) e aos observadores das estações fluviométricas: Pedro Paulo dos Santos, Maria Augusta Coelho, Osvaldo da Silva, Adão Valter Parolin, Hugo Selmiro Rodher, Jatir Maronese, Eliezer Marcos Brustolin, Edson Nascimento Dias, João Ferreira e Aguiomar José Jaiewky, pelo auxílio nas atividades de campo.

\section{RELAÇÕES PRELIMINARES DA GEOMETRIA HIDRÁULICA REGIONAL PARA RIOS DAS REGIÔES OESTE E SUDOESTE DO ESTADO DO PARANÁ}

Resumo: O trabalho apresenta os resultados preliminares das relações da geometria hidráulica regional para rios das regiões Oeste e Sudoeste do Estado do Paraná. A cota dos diques marginais foi adotada como referencia na definição do nível de margens plenas. A área de drenagem das estações variou de 969 a $12.124 \mathrm{~km}^{2}$. As relações entre a área de drenagem e a vazão, largura, profundidade e capacidade do canal, todas medidas em nível de margens plenas, alcançaram valores de coeficiente de correlação $\left(\mathrm{R}^{2}\right)$ iguais a 0,$94 ; 0,89 ; 0,49$ e 0,83 respectivamente. Estes resultados mostram a forte correlação entre as variáveis da geometria hidráulica regional para as estações fluviométricas inseridas numa região fisiográfica caracterizada pela homogeneidade climática e geológica. As equações permitem estimar a vazão e as dimensões do canal em nível de margens plenas nas bacias em apreço, a partir da área da bacia. As informações obtidas podem orientar técnicos em projetos de restauração de canais e direcionar trabalhos de avaliação das condições de degradação em ambientes fluviais

Palavras-chave: geomorfologia fluvial; geometria hidráulica; descarga de margens plenas. 
Abstract: This paper presents the preliminary results of hydraulic geometry relations for rivers of the Western and Southwestern regions of Paraná Sate, Brazil. The natural levee was adopted as reference in determining the bankfull stage. The drainage area of the gauging stations ranged from 969 to $12,124 \mathrm{~km}^{2}$. The relationship between drainage area and discharge, width, depth and channel capacity, all measures at bankfull stage, reaching values of correlation coefficient $\left(\mathrm{R}^{2}\right)$ equal to $0.94,0.89$, 0.49 and 0.83 respectively. These results show a strong correlation between the variables of regional hydraulic geometry for the gauged stations included in a physiographic region characterized by uniform climatic and geological conditions. The equations to estimate discharge and dimensions of the channel at bankfull stage from the watershed area. The information obtained can drive technical projects for river restoration and direct evaluations of the conditions of degradation in river environments

Key words: fluvial geomorphology; hydraulic geometry; bankfull discharge.

\section{BIBLIOGRAFIA}

ALMEIDA, F.F.M. de (1966) Origem e evolução da Plataforma Brasileira. Sedegeo, 2: 46-89

ANDREWS, E.D. (1980) Effective and bankfull discharges of streams in the Yampa River Basin, Colorado and Wyoming. Journal of Hydrology, 46: 311-330.

AQUINO, S.; STEVAUX, J.C.; LATRUBESSE, E.M. (2005) Regime hidrológico e aspectos do comportamento morfohidráulico do rio Araguaia. Revista Brasileira de Geomorfologia, 6 (2): 29-41.

BENSON, M.A.; THOMAS, D.M. (1966) A definition of dominant discharge. Bulletin of the International Association of Scientific Hydrology, 11: 76-80.

BRAY, D.I. (1972) Generalized regime-type analysis of Alberta rivers. Ph.D thesis, University of Alberta, Edmonton, Canada

CARLING, P. (1988) The concept of dominant discharge applied to two gravel-bed streams in relation to channel stability thresholds. Earth Surface Processes and Landforms, 13: 355-367. 
CASTRO, J.M.; JACKSON, P.C. (2001) Bankfull discharge recurrence intervals and regional hydraulic geometry relationships: patterns in the Pacific Northwest, USA. Journal of the American Water Resources Association, 37 (5): 1249-1262.

CHISTOFOLETTI, A. (1976) Geometria hidráulica. Notícias Geomorfológicas, 16 (32):3-37.

CHISTOFOLETTI, A. (1981) Geomorfologia Fluvial. São Paulo: Editora E. Blücher Ltda., 313 p.

CINOTTO, P. J. (2003) Development of regional curves of bankfullchannel geometry and discharge for streams in the non-urban, Piedmont physiographic province, Pennsylvania and Maryland. USGS WaterResources Investigations Report 03-4014.

DUNNE, T.; LEOPOLD, L.B. (1978) Water in Environmental Planning. San Francisco: W.H. Freeman Co.

DURY, G.H. (1973) Magnitude-frequency analysis and channel morphology. In: Morisawa, M. (Org.) Fluvial Geomorphology. Allen and Unwin, 91-121

DURY, G.H. (1976) Discharge prediction, present and former from channel dimensions. Journal of Hydrology, 30: 219-245.

EMMERT, B.A. (2004) Regional curve development for Kansas. Proceedings of the ASAE September Conference: Self-Sustaining Solutions for Streams, Wetlands and Watersheds. September 12-15, 2004. St. Paul, Minnesota: 27-35.

EMMETT, W.W. (1975) The channels and waters of the Upper Salmon River area, Idaho. U.S. Geological Survey Professional Paper, 870-A 116 p.

EMMETT, W.W.; WOLMAN, M.G. (2001) Effective discharge and gravel-bed rivers. Earth Surface Processes and Landform, 26: 1369-1380.

FERGUSON, R.I. (1986) Hydraulic and hydrologic geometry. Progress in Physical Geography, 10: 1-31.

FERNANDEZ, O.V.Q.; BORTOLUZZI, L.N. (2008) Magnitude e frequiência das descargas dominantes em rios das regiões Oeste e Sudoeste do Paraná. Anais... VII Simpósio Nacional de Geomorfologia, Belo Horizonte (MG), UFMG, 11 p., CD-Rom.

HARMAN, W.A.; JENNINGS, G.; PATTERSON, J.; CLINTON, D., SLATE, L., JESSUP, A., EVERHART, J. E SMITH, R. (1999) Bankfull hydraulic geometry relationships for North Carolina streams. In: AWRA 
Wildland Hydrology Symposium Proceedings (D. Olsen \& J. Potyondy, Eds.), Bozeman, Montana, 401-408.

HARVEY, A.M. (1969) Channel capacity and the adjustment of streams to hydrologic regime. Journal of Hydrology, 8: 82-98.

HICKIN, E.J. (1968) Channel morphology bankfull stage and bankfull discharge of streams near Sydney, Australia. Journal of Science, 30 (7): 274-275.

IAPAR - Fundação Instituto Agronômico do Paraná (1994) Cartas climáticas básicas do Estado do Paraná, Curitiba (PR), 49 p.

INGLIS, C.C. (1949) The behavior and control of rivers and canals. Research Publication 13, Central Waterpower Irrigation and Navigation Research Station, Poona, India, p. 79-91.

KEATON, J. N., MESSINGER, T.; DOHENY, E.J. (2005) Development and analysis of regional curves for streams in the non-urban valley and Ridge physiographic province, Maryland, Virginia, and West Virginia. Scientific Investigations Report 2005-5076. USGS, 116 pp.

KELLERHALS, R. NEILS, C.R.; BRAY, D.I. (1972) Hydraulic and geomorphic characteristics of rivers in Alberta. Edmonton: Resources Council of Alberta. $52 \mathrm{p}$.

KILPATRICK, F.A.; BARNES Jr., H.H. (1964) Channel geometry of Piedmont streams as related to frequency of floods. U.S. Geological Survey

KNIGHTON, A.D. (1975) Variations in at-a-station hydraulic geometry. American Journal of Science, 275: 186-218.

LATRUBESSE, E.M.; AQUINO, S. (1998) Geometria hidráulica em rios da Amazônia Sul-Ocidental. Revista Geosul, Florianópolis, UFSC, 14 (27): 610-613.

LEOPOLD, L.B. (1994) A view of the river. Cambridge, Mass.: Harvard University Press.

LEOPOLD, L.B.; MADDOCK, T. (1953) The hydraulic geometry of stream channels and some physiographic implications. Professional Paper, United States Geological Survey, Washington, 252: 57 p.

LEOPOLD, L.B.; SKIBITZKE, H.E. (1967) Observation on unmeasured rivers. Geographical Annaler 49: 247-255.

LEWIS, C.P.; MCDONALD, B.C. (1973) Rivers of the Yukon north slope. In: Fluvial Processes and Sedimentation, pp. 251-271.

McCANDLESS, T.L. (2003) Maryland stream survey: bankfull discharge and channel characteristics of streams in the Allegheny plateau and the 
valley and ridge hydrologic regions. Annapolis: U.S. Fish and Wildlife Service, Chesapeake Bay Field Office, 33p.

MESSINGER, T.; WILEY, J.B. (2003) Regional relations in bankfull channel characteristics determined from flow measurements at selected stream-gauging stations in West Virginia, 1911-2002. 03-4276. USGS. 43 pp.

MONTGOMERY, D.R.; GRAN, K.B. (2001) Downstream variations in the width of bedrock channels. Water Resources Research 37 (6): 18411846.

MOODY, T. O.; ODEM, W. (1999). Regional relationships for bankfull stage in natural channels of Central and Southern Arizona. Phoenix: Arizona Department of Environmental Quality.

NARDY, A.J.R.; OLIVEIRA, M.A.F.; BETANCOURT, R.H.S.; VERDUGO, D.R.H.; MACHADO, F.B. (2002) Geologia e estratigrafia da Formação Serra Geral. Geociências (Unesp, São Paulo): 21: 15-32

NASH, D.B. (1994) Effective sediment-transporting discharge from magnitude-frequency analysis. Journal of Geology, 102: 79-95.

NAVRATIL, O.; ALBERT, M.B.; HÉROUIN, E.; GRESILLLON, J.M. (2006) Determination of bankfull discharge magnitude and frequency: comparison of methods on 16 gravel-bed river reaches. Earth Surface Processes and Landforms 31: 1345-1363.

NIXON, M. (1959) A study of bankfull discharge of rivers in England and Wales. Proceedings of the Institution of Civil Engineers, 12: 157-175.

NUNNALLY, N.R. (1967) Definition and identification of channel and overbank deposits and their respective roles in floodplain formation. Professional Geographer 19: 1-4.

PARK, C.C. (1977) World-wide variations in hydraulic geometry exponents of stream channels: an analysis and some observations. Journal of Hydrology, 33: 133-146.

PICKUP, G.; WARNER, R.F. (1976) Effects of hydrologic regime on magnitude and frequency of dominant discharge. Journal of Hydrology, 29: 51-75.

PICKUP, G.; RIEGER, W.A. (1979) A conceptual model of the relationship between channel characteristics and discharge. Earth Surface Processes, 4: 37-42.

POWELL, R.O., MILLER, S.J., WESTERGARD, B.E., MULVIHILL, C.I., BALDIGO, B.P., GALLAGHER, A.S. e STARR, R.R. (2004) Guidelines for surveying bankfull channel geometry and developing 
regional hydraulic-geometry relations for streams of New York State. USGS 03-92. 20 pp.

ROSGEN, D.L. (1994) A classification of natural rivers. Catena 22: 169199.

RADECKI-PAWLIK, A. (2002) Bankfull discharge in mountain streams: theory and practice. Earth Surface Processes and Landforms, 27: 115-123.

RILEY, S.J. (1972) A comparison of morphometric measures of bankfull. Journal of Hydrology, 17: 23-31.

RILEY, S.J. (1976) Aspects of bankfull geometry in a distributary system of eastern Australia. Hydrological Sciences Journal, 21: 545-560.

RHOADS, B.L. (1992) Statistical models of fluvial systems. Geomorphology, 5: 433-455.

SCHUMM, S.A. (1960) The shape of alluvial channels in relation to sediment type. U.S. Geological Survey Professional Paper 352.

SHERWOOD, J.M.; HUITGER, C.A. (2005) Bankfull characteristics of Ohio streams and their relation to peak stream-flows. U.S. Geological Survey Scientific Investigations Report 2005-5153, 38 p.

SWEET, W.V.; GERATZ, J.W. (2003) Bankfull hydraulic geometry relationships and recurrence intervals for North Carolina's coastal plain. Journal of the American Water Resources Association, 39(4):861-871.

THORNES, J.B. (1977) Hydraulic geometry and channels change. In: K.J. Gregory (Ed.), River Channels Changes, Wiley, Chichester: p. 91-100.

WHITE, K. E. (2001) Regional curve development and section of a reference reach in the non-urban, lowland sections of the Piedmont physiographic province, Pennsylvania and Maryland. USGS.

WHITING, P. J., STAMM, J.F.; MOOG, D.B.; ORNDOFF, R.L. (1999). Sediment-transporting flows in headwater streams. GSA Bulletin 111, p. 450-466.

WILLIAMS, G.P. (1978) Bank-full discharge of rivers. Water Resources Research, 14 (6): 1141-1153.

WOHL, E., KUZMA, J., BROWN, N.E. (2004) Reach-scale channel geometry of a mountain river. Earth Surface Processes and Landforms 29: 969-981.

WOHL, E., WILCOX, A. (2005) Channel geometry of mountain stream in New Zealand. Journal of Hydrology 300: 255-266.

WOLMAN, M.G. (1955) The natural channel of Brandywine creek. Pennsylvania. U.S. Geological Survey Professional Paper, 271, 56 p 
WOLMAN, M.G.; LEOPOLD, L.B. (1957) River flood plains: some observations on their formation. Profissional Paper, United States Geological Survey, 282C, p. 87-107.

WOODYER, K.D. (1968) Bankfull frequency in rivers. Journal of Hydrology 6: 114-142. 